\title{
The Utility of MEWS for Predicting the Mortality in the Elderly Adults with COVID-19: A Retrospective Cohort Study with Comparison to Other Predictive Clinical Scores
}

\author{
Lichun Wang Equal first author, 1, Qingquan Lv ${ }^{\text {Equal first author, } 2}{ }^{\text {, Xiaofei Zhang }}{ }^{1}$, Binyan Jiang ${ }^{3}$, Enhe Liu ${ }^{4}$, Chaoxing Xiao ${ }^{4}$, \\ Xinyang Yu ${ }^{3}$, Chunhua Yang ${ }^{1}$, Lei Chen ${ }^{\text {Corresp. } 1}$ \\ ${ }^{1}$ Department of Critical Care Medicine, The Sixth Affiliated Hospital of Sun Yat-Sen University, guangzhou, guangdong, China \\ 2 Department of health services section,, Wuhan Hankou hospital, wuhan, hubei, China \\ 3 Department of Applied Mathematics, The Hong Kong Polytechnic University, hongkong, China \\ 4 Department of Critical Care Medicine, Foresea Life Insurance Guangzhou General Hospital, guangzhou, China \\ Corresponding Author: Lei Chen \\ Email address: chenlei6@mail.sysu.edu.cn
}

Background: Older adults have been reported to be a population with high-risk of death in the COVID-19 outbreak. Rapid detection of high-risk patients is crucial to reduce mortality in this population. The aim of this study was to evaluate the prognositc accuracy of Modified Early Warning Score (MEWS) for in-hospital mortality in older adults with COVID-19. Methods A retrospective cohort study was conducted in Wuhan Hankou Hospital in China from 1 January 2020 to 29 February 2020. Receiver operating characteristic (ROC) analysis was used to evaluate the predictive value of MEWS $\square$ Acute Physiology and Chronic Health Evaluation II (APACHE II), Sequential Organ Function Assessment (SOFA), quick Sequential Organ Function Assessment (qSOFA), Pneumonia Severity Index (PSI), Combination of Confusion, Urea, Respiratory Rate, Blood Pressure, and Age $\geq 65$ (CURB-65), and the Systemic Inflammatory Response Syndrome Criteria (SIRS) for in-hospital mortality. Logistic regression models were performed to detect the high-risk older adults with COVID-19. Results: Among the 235 patients included in this study, 37 (15.74\%) died and 131 (55.74\%) were male, with an average age of 70.61 years (SD 8.02). ROC analysis suggested that the capacity of MEWS in predicting in-hospital mortality was as good as the APACHE II, SOFA, PSI and qSOFA (Difference in AUROC: MEWS VS APACHE II, -0.025 [95\% Cl, $(-0.075,0.026)]$; MEWS VS SOFA, -0.013 [95\% Cl, $(-0.049$, 0.024)]; MEWS VS PSI, -0.015 [95\% Cl, $(-0.065,0.035)$ ]; MEWS VS qSOFA, 0.024 [95\% Cl, $(-0.029,0.076)]$, all $P \square 0.05)$, but was significantly higher than SIRS and CURB-65 (Difference in AUROC: MEWS VS SIRS, 0.218 [95\% Cl, 0.156,0.279)]; MEWS VS CURB-65, $0.064[95 \% \mathrm{Cl},(0.002,0.125)]$, all P $\square 0.05)$. Logistic regression models implied that the male patients ( $\geq 75$ years) had higher risk of death than the other older adults (estimated

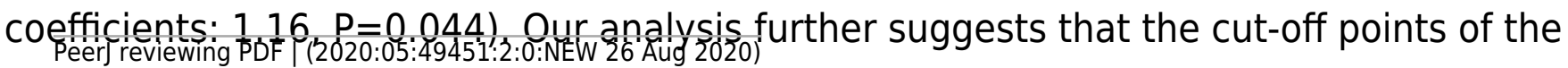


MEWS score for the male patients ( $\geq 75$ years) subpopulation and the other elderly patients should be 2.5 and 3.5, respectively. Conclusions: MEWS is an efficient tool for rapid assessment of elderly COVID-19 patients. MEWS has promising performance in predicting in-hospital mortality and identifying the high-risk group in elderly patients with COVID-19. 
1 The Utility of MEWS for Predicting the Mortality in the Elderly Adults with COVID-19: A

3 LiChun Wang ${ }^{1}$, Qingquan Lv², Xiaofei Zhang ${ }^{1}$, Binyan Jiang ${ }^{3}$, Enhe Liu ${ }^{4}$, Chaoxing Xiao ${ }^{4}$,

4 Xinyang $\mathrm{Yu}^{3}$, Chunhua Yang ${ }^{1}$, Lei $\mathrm{Chen}^{1}$.

\section{Author Affiliations:}

$6 \quad{ }^{1}$ Department of Critical Care Medicine, The Sixth Affiliated Hospital of Sun Yat-Sen

7 University, Guangzhou, Guangdong, China;

$8{ }^{2}$ Department of health services section, Wuhan Hankou hospital, Wuhan, Hubei, China;

$9{ }^{3}$ Department of Applied Mathematics, The Hong Kong Polytechnic University, Hong Kong,

10 China;

$11{ }^{4}$ Department of Critical Care Medicine, Foresea Life Insurance Guangzhou General Hospital,

12 Guangzhou, Guangdong, China.

13 Lichun Wang and Qingquan Lv contributed equally and share first authorship.

\section{Corresponding author:}

15 Lei Chen

16 Department of Intensive Care Unit, The Sixth Affiliated Hospital of Sun Yat-Sen University,

17 Guangzhou, Guangdong, China.

18 E-mail: chenlei6@mail.sysu.edu 
The Utility of MEWS for Predicting the Outcome in the Elderly Adults with COVID-19: A

\section{Retrospective Cohort Study with Comparison to Other Predictive Clinical Scores}

Background: Older adults have been reported to be a population with high-risk of death in the COVID-19 outbreak. Rapid detection of high-risk patients is crucial to reduce mortality in this population. The aim of this study was to evaluate the prognostic accuracy of Modified Early Warning Score (MEWS) for in-hospital mortality in older adults with COVID-19.

Methods A retrospective cohort study was conducted in Wuhan Hankou Hospital in China from 1 January 2020 to 29 February 2020. Receiver operating characteristic (ROC) analysis was used to evaluate the predictive value of MEWS, Acute Physiology and Chronic Health Evaluation II (APACHE II), Sequential Organ Function Assessment (SOFA), quick Sequential Organ Function Assessment (qSOFA), Pneumonia Severity Index (PSI), Combination of Confusion, Urea, Respiratory Rate, Blood Pressure, and Age $\geq 65$ (CURB-65), and the Systemic Inflammatory Response Syndrome Criteria (SIRS) for in-hospital mortality. Logistic regression models were performed to detect the high-risk older adults with COVID-19.

Results: Among the 235 patients included in this study, 37 (15.74\%) died and 131 (55.74\%) were male, with an average age of 70.61 years (SD 8.02). ROC analysis suggested that the capacity of MEWS in predicting in-hospital mortality was as good as the APACHE II, SOFA, PSI and qSOFA (Difference in AUROC: MEWS VS APACHE II , -0.025 [95\% CI, (-0.075, 0.026)]; MEWS VS SOFA, -0.013 [95\% CI, (-0.049, 0.024)]; MEWS VS PSI, -0.015 [95\% CI, $(-0.065,0.035)]$; MEWS VS qSOFA, $0.024[95 \% \mathrm{CI},(-0.029,0.076)]$, all $\mathrm{P} \square 0.05)$, but was significantly higher than SIRS and CURB-65 (Difference in AUROC: MEWS VS SIRS, 0.218 
$41 \quad[95 \%$ CI, 0.156,0.279)]; MEWS VS CURB-65, 0.064 [95\% CI, (0.002, 0.125)], all P $\square 0.05)$.

42 Logistic regression models implied that the male patients ( $\geq 75$ years) had higher risk of death

43 than the other older adults (estimated coefficients: $1.16, \mathrm{P}=0.044$ ). Our analysis further suggests

44 that the cut-off points of the MEWS score for the male patients ( $\geq 75$ years) subpopulation and

45 the other elderly patients should be 2.5 and 3.5 , respectively.

46 Conclusions: MEWS is an efficient tool for rapid assessment of elderly COVID-19 patients.

47 MEWS has promising performance in predicting in-hospital mortality and identifying the high-

48 risk group in elderly patients with COVID-19.

49 Key words: COVID-19; Modified Early Warning Score; Older Adults; Outcome. 
INTRODUCTION

52 The WHO Director-General declared COVID-19 as a pandemic on $11^{\text {th }}$ March, 2020. According

53 to the report of WHO-China Joint Mission on Coronavirus Disease 2019 (COVID-19)

54 Report,(Wang et al. 2019) the older adults who are above 60 years old are the population with

55 high-risk of death. The crude mortality of the older adults above 80 years old could be as high as

$5621.9 \%$. An epidemiological investigation conducted by the Chinese Center for Disease Control

57 and Prevention found that more than one third of the COVID-19 patients were the older adults

58 aged 60 years and above. (Wu \& McGoogan 2020)

59 While older adults have been identified as the main group with COVID-19 infection, the vital

60 signs of these patients could deteriorate very quickly. Hence timely detection of the high-risk

61 group and appropriately-assessed disease severity for the older adults with COVID-19 are

62 practically essential.(Verity et al. 2020) Several disease severity scoring systems are often used

63 to guide the management of patients with pneumonia, such as Acute Physiology and Chronic

64 Health Evaluation II (APACHE II), Sequential Organ Function Assessment (SOFA), quick

65 Sequential Organ Function Assessment (qSOFA), Pneumonia Severity Index (PSI), Combination

66 of Confusion, Urea, Respiratory Rate, Blood Pressure, and Age $\geq 65$ (CURB-65), Modified Early

67 Warning Score (MEWS) and the Systemic Inflammatory Response Syndrome Criteria

68 (SIRS).(Asai et al. 2019; Chalmers et al. 2011; Dremsizov et al. 2006; Goodacre et al. 2010; Kim

69 et al. 2019; Sikka et al. 2000) All these scoring systems have been proven to be useful for

70 predicting the outcome of patient's with pneumonia.

71 Among these scoring systems, MEWS can generally be obtained within minutes after the

Peer] reviewing PDF | (2020:05:49451:2:0:NEW 26 Aug 2020) 
72

patient is admitted, providing a rapid assessment result for clinicians and enabling timely treatment to high risk patients.(Churpek et al. 2019; Salottolo et al. 2017; Yu et al. 2019) Although MEWS has been found to be useful for assessment of pneumonia deterioration,(Jo et al. 2016) its utility has not been examined in predicting outcome of the older adults with COVID-19. The aim of this study is to evaluate the prognostic accuracy of MEWS for inhospital mortality of older adults with with COVID-19.

\section{METHODS}

\section{Study Population}

This is a retrospective cohort study conducted in the Wuhan Hankou Hospital in China from 1 January 2020 to 29 February 2020. It was approved by the Institutional Ethics Committee of Hankou hospital (hkyy2020-014). The Institutional Ethics Committee approved of waiving informed consents by reasons that this study was conducted retrospectively, privacy and personally identifiable information of the patients were protected and data collection would not harm the patient. The data in this study were analyzed anonymously. Inclusion criteria were as follows: (1) (1) Patients diagnosed as COVID-19 (Supplementary Text S1); (2) Patients treated by Guangdong medical team; (3) Older adults aged 60 or above. Exclusion criteria: (1) pneumonia caused by other pathogens; (2) incomplete data.

\section{Clinical and Biochemical Data Collection}

92 All the data were obtained from the electronic medical records in the Information System of 
93

94

95

Wuhan Hankou Hospital. The information of the patients during admission including age, gender, underlying disease, consciousness state, vital signs, physiological and laboratory variables were collected and further used for the calculation of APACH II score, PSI score, SOFA score, qSOFA score and MEWS (Supplementary Text S2). All these variables/factors are objective measurable parameters collected by professional medical staff, and the laboratory variables were recorded and double check by two researchers.

\section{Outcomes variables}

The main outcome of the study was in-hospital mortality. The other issue we tried to address was to identify the group of the high-risk patients based on MEWS. Patients were further categorized into different groups by their gender (male VS female) and age ( 75 years old or above VS 60-74 years old).

\section{Statistical Analysis}

Statistical analysis was carried out by Microsoft R (version 3.5.3). All the disease severity scores were described as Median (Inter-Quartile Range), while other numerical variables were described as Mean (SD). Categorical variables were reported as percentages and frequencies. Kolmogorov-Smirnov test was used to assess the normality of the numerical variables, and subsequently, we used T-test to compare normally distributed variables and used Mann-Whitney test to test whether the means between two groups are significantly different for non-normally distributed variables. Chi-square test was used to test independence for contingency tables of 
114 categorical variables. Receiver Operating Characteristic (ROC) curve was used to evaluate the

115 predictive value of each scoring system for prognosis, and Z-test was used for the Area Under

116 the ROC curve (AUROC). In order to evaluate the accuracy of each scoring system sensitivity,

117 specificity, positive predictive value and negative predictive value were determined. Optimal

118 cut-off value for each score was calculated using Youden's J-statistics. Bootstrapping confidence

119 intervals were calculated to evaluate the difference in the AUROC between MEWS and the other

120 scores. Specifically, by resampling (with replacement) the data for 100 times, we obtained an

121 empirical distribution for the difference of AUROC between MEWS and other scores. The

confidence intervals were then obtained using the percentiles of the empirical distribution. In

order to detect the high-risk population and understand the risk effect of the MEWS on the

mortality rate in different subpopulations, logistic regression models were developed against

MEWS, age groups, gender and the interaction between age group and gender. The optimal

model was selected by Akaike Information Criterion (AIC). A smaller AIC score indicates a

better fit of the corresponding model to the data. All the hypothesis tests were 2-sided with a significance level of 0.05 .

\section{RESULTS}

\section{Study Population and outcomes}

The total number of patients treated by Guangdong medical team was 472, among which 244

$133(51.7 \%)$ met the enrollment criteria (patients aged 60 years old or above with a primary diagnosis of COVID-19 disease) from the 1 January 2020 to 29 February 2020. 9 patients were excluded 
135 from our study cause 1 patient had been diagnosed with pneumonia caused by other pathogens

136 and 8 patients had incomplete data. Therefore, there were 235 patients included in this analysis.

137 The general information and the clinical characteristics of the patients are listed in Table 1.

138 The mean age was 70.6 years (SD 8.0 ) and $55.7 \%$ of them were male. $43.8 \%$ of these patients

139 had Hypertension, 26.4\% of them had Diabetic Mellitus, and 20.9\% had history of Coronary

140 Heart Disease. The median values of scores in these patients were as follows: 12 (IQR: 9-17) in

141 APACHE II score, 3 (IQR: 2-5) in SOFA score, 82 (IQR: 65-114) in PSI score, 1 (IQR: 1-2) in

142 CURB-65, 2 (IQR: 1-4) in MEWS and 0 (IQR: 0-1) in qSOFA. 56.2\% of these patients met

143 SIRS criteria (Table 1).

144 Non-survivors were more likely to be male (27 [73.0\%] VS 104 [52.5\%]), with higher

145

146

147

148

149

150

151

152

153

154 155

APACHE II score (24 [IQR: 20-27] VS 11 [IQR: 9-14]), SOFA score (7 [IQR: 6-9] VS 3 [IQR:

2-4]), PSI score (152 [IQR: 128-166] VS 75[IQR: 63-100]), CURB-65 score (3 [IQR: 2-4] VS

1[IQR: 1-2]), MEWS score (5 [IQR: 4-6] VS 2[IQR: 1-3])and qSOFA score (2 [IQR: 1-2] VS 0

[IQR: 0-11]) than survivors. In addition, more non-survivors met SIRS criteria (33[89.2\%] VS

$99[50.0 \%])$.

Of the 235 patients during follow-up, 37 (15.8\%) died in the hospital and the median duration of hospital stay of the non-survivors were 5.5 days (IQR: 2.0-9.3).

\section{Prognostic accuracy of MEWS in predicting the in-hospital mortality rate}

54 The distributions of all the scores were presented at Figure S1, and their relationships with inhospital mortality in the older adults with COVID-19 were presented in Figure 1. The AUROCs 
156 for these scores in predicting in-hospital mortality were as follows: APACHE $\mathbb{I}, 0.937$ [95\% CI, 157 (0.877, 0.995)]; SOFA, 0.926 [95\% CI, (0.877, 0.975)]; PSI, 0.927 [95\% CI, (0.898, 0.986)];

158

159

160

161

162

163

164

165

166

167

168

169

170

171

172

173

174

175

176

CURB-65, 0.845 [95\% CI, (0.740, 0.951)]; MEWS, 0.913 [95\% CI, (0.864,0.941)]; SIRS, 0.696

[95\% CI, (0.616, 0.776)]; and qSOFA, .886 [95\% CI, (0.804, 0.969)] (Table 2 and Figure 2).

The reliability of MEWS in predicting in-hospital mortality was as good as the APACHE II score, SOFA score, PSI score and qSOFA (Difference in AUROC with MEWS: MEWS VS

APACHE II , -0.025 [95\% CI, (-0.075, 0.026), P=0.828]; MEWS VS PSI, -0.013 [95\% CI, (0.049, 0.024), $\mathrm{P}=0.748]$; MEWS VS PSI, -0.015 [95\% CI, $(-0.065,0.035), \mathrm{P}=0.735]$; MEWS VS qSOFA, $0.024[95 \% \mathrm{CI},(-0.029,0.076), \mathrm{P}=0.174]$, but the prognostic accuracy of MEWS was significantly higher as compared with either SIRS criteria or CURB-65 score (Difference in AUROC with MEWS: MEWS VS SIRS, 0.218 [95\% CI, $(0.156, .279), \mathrm{P}<0.001]$; MEWS VS CURB-65, 0.064 [95\% CI, $(0.002,0.125), \mathrm{P}=0.015])$. The optimal cutoff value of MEWS for predicting the inpatient mortality in the older adults with COVID-19 was 4.5 , with a specificity of $94.5 \%$ and a sensitivity of $67.6 \%$.

We also analysed the age adjusted ROC curves and AUROCs to evaluate the prognostic accuracy of MEWS).. However, no significant difference was observed from the original analysis without age adjustment (Table 1).

\section{Identifying the risk population with high mortality via logistic regression models}

In order to identify the high-risk older adults with COVID-19 and understand the prognostic accuracy of the MEWS on the mortality in different subpopulations categorized by gender and 
177 age group, logistic regression models were developed. In particular, an interaction term

178

179

180

181

182

183

184

185

186

187

age*gender term was introduced to consider possible interaction effect between age group and gender. The optimal model was selected by AIC. The best model is the one with features age*gender (estimated coefficients: 1.16, $\mathrm{P}=0.044)+\mathrm{MEWS}$ (estimated coefficients: 1.24, $\mathrm{P}<0.001$ ) (Table 3). Note that under our notations, the age*gender indicator corresponds to whether the patient is a male patient aged 75 years old or above. The positive coefficient (1.16) thus implies the group of COVID-19 patients who were male and aged 75 years or above had higher risk of death than the other COVID-19 patients in this population. In particular, since the coefficient of age*gender (1.16) is very close to the coefficient of the MEWS (1.24), i.e., $1.16 / 1.24 \approx 1$, the risk of male COVID-19 patients aged 75 years and above for a given MEWS is as high as the risk of other patients with a one mark higher MEWS score. Motivated by this, we further explore the risk effect of the MEWS in the two subpopulations (male patients aged 75 years or above VS other patients in this population) separately. Results on the prognostic accuracy of MEWS in predicting the in-hospital mortality of these two subpopulations were showed in Supplementary Table S1 and Figure S2. The optimal cut-off value of MEWS in the male COVID-19 patients aged 75 years or above was 2.5 , with $84.3 \%$ specificity, $84.6 \%$ sensitivity, $57.9 \%$ mortality rate for those with MEWS $>2.5$, and $0.0 \%$ mortality rate for those with MEWS<2.5. While for other older adults with COVID-19, the optimal cut-off value of MEWS was 3.5, with $75.0 \%$ specificity, $100 \%$ sensitivity, $45.8 \%$ mortality rate for those with MEWS $>3.5$, and $2.8 \%$ mortality rate for those with MEWS $<3.5$. We remark that there is a one point difference in the cut-off points of the two subpopulations (2.5 VS 3.5). This is consistent to 
198

199

200

201

202

203

204

205

206

207

208

209

210

211

212

213

214

215

216

217

218

the results we have discussed for the fitted model.

\section{DISCUSSION}

In this retrospective cohort study, ROC analysis suggested that prognostic accuracy of MEWS in predicting the inpatient mortality rate is comparable to that of APACHE $I$ score, PSI score, SOFA score, and better than the CURB-65, SIRS criteria in older adults with COVID-19. On the other hand, comparing to APACHE II, PSI and SOFA, MEWS is much simpler and can be rapidly obtained in clinical practice. Our analysis has also shown MEWS to be a promising tool for risk stratification of these patients. We thus conclude that MEWS is an effective prognostic tool for providing rapid assessment and identifying the high-risk patients among the older adults with COVID-19, so the use of MEWS should be encouraged in this population during this severe plague outbreak.

Since the outbreak of COVID-19 has begun, as of July 103, 2020, more than 12 million cases over the world have been confirmed and over half million deaths have occurred. COVID-19 has resulted in considerable morbidity and mortality in more than 200 countries worldwide. There is no specific drug for treating the patients with COVID-19, till mow treatment is mainly symptomatic. Recent literatures have identified older adults group are more prone towards COVID-19 infection, and they might be at higher risk of death than younger patients.(Liu et al. 2020; Onder et al. 2020; Verity et al. 2020; Wu \& McGoogan 2020) It is reported that the mortality in the patients aged 60 years or above ranged from $5.98 \%$ to $12.4 \%$, while the mortality in the younger patients ranged from $1.32 \%$ to $0.64 \%$.(Onder et al. 2020; Wu \& McGoogan 2020) 
219 In our study, mortality in older adults with COVID-19 aged 60 years or above is $15.8 \%$. A recent

220 study demonstrated that older adults aged 65 years or above with COVID-19 are more likely to

221 develop organ injury in a short time, leading to an increased risk of death.(Yang et al. 2020) In

222 our study, we found that the median duration of hospital stay of the non-survivors was 5.5 days

223 (IQR: 2.0-9.3), indicating that the vital signs of these patients could deteriorate very quickly.

224 Early identification of the high-risk patients and timely medical interventions could possibly

225

226

227

228

229

230

231

232

233

234

235

236

237

238

239

reduce the high mortality rate among this population. Rapid and effective assessment of these

older adults with COVID-19 is thus very crucial. Owning to the rapid spread of COVID-19,

there has been a severe shortage of medical resources, so rapid assessment could potentially

serve as an essential tool for efficient allocation of these limited resources to those who are

indeed at has been high risk of death.

PSI score and CURB-65 score have been wildly used for prognostic evaluations and severity

assessment of pneumonia, which enables the clinicians to allocate the patients to appropriate

level and intensity of care and perform effective medical management in time.(Kim et al. 2019;

Ning et al. 2018) It had reported that high PSI score, CURB-65 score, APACHE II score and

SOFA score were associated with poor outcome in the patients with pneumonia.(Asai et al. 2019;

Kao et al. 2018) Since the outbreak of COVID-19, APACHE II score, CURB-65 score, SOFA score and qSOFA score have been used to assess the severity of COVID-19 patients.(Wang et al. 2020; Yang et al. 2020; Zhou et al. 2020) In a cohort of 52 patients with COVID 19, nonsurvivors had higher APACHE $\mathbb{I}$ score than the survivors (18 [IQR: 16-20] VS 14 [IQR : 1217]).(Yang et al. 2020) A recent study conducted by Fei Zhou(Zhou et al. 2020) indicated that 
240

241

242

243

244

245

246

247

248

249

250

251

252

253

254

255

256

257

258

259

260

non-survivors had higher SOFA score (4·5 [IQR: 4.0-6.0] VS 1.0 [IQR: 1.0-2.0], P<·0001),

qSOFA score (1 [IQR: 1.0-2.0] VS 0 [IQR: 0.00-1.0], P<.0001), CURB-65 (2.0 [IQR: 1.0-3.0]

VS 1.0 [IQR:0.0-1.0], P $<0.0001$ ) than survivors. It is worth mentioning that, non-survivors in the older adult COVID-19 population we studied had higher APACHE II score (24 [IQR: 2027]), SOFA score (7 [IQR: 6.0-9.0]), CURB-65 score (3 [IQR: 2.0-4.0]) and qSOFA score (2 [IQR: 1.0-2.0]), compared to previous studies with different populations. Although APACHE II, SOFA, PSI and CURB-65 work well in clinical practice, they generally require sophisticated information from the patient and are not suitable for rapid assessment.

MEWS consists of 5 physiological parameters, which include systolic blood pressure, pulse rate, respiratory rate, temperature, and level of consciousness. It has been used as an essential tool for early identification of patients who can deteriorate in medical and surgical wards.(Fullerton et al. 2012; Kramer et al. 2019; Salottolo et al. 2017) Some studies have shown that a MEWS score of 5 or above is associated with a high risk of poor outcome in intensive care unit.(Reini et al. 2012) Comparing with other scores, MEWS is much simpler and more importantly allows rapid assessment and dynamic monitoring. However, the capacity of MEWS on COVID-19 patients hasn't been well explored yet. This retrospective cohort study focused on the utility of MEWS in predicting outcome in the older adults with COVID-19. We observed that, for the older adults with COVID-19, the capacity of MEWS to predict in-hospital mortality was significantly higher as compared with SIRS criteria and CURB-65 score, while the prognostic accuracy is comparable to those of APACHE $\amalg$, PSI and SOFA. More importantly, MEWS allows early detection of the high-risk older adults with COVID-19. We found that the 
261

262

263

264

265

266

267

268

269

270

271

272

273

274

275

276

277

278

279

280

281

cut-off values using MEWS to predict the outcome in COVID-19 is smaller than the 5 points in the guidelines for other disease. In addition, the present study reveals that the male COVID-19 patients aged 75 years or above had higher risk of death than the other older adults with COVID19. In particular, the cut-off points of the MEWS for the male COVID-19 patients aged 75 years or above should be one-point lower than the cut-off for the other older adults with COVID-19 (2.5 VS 3.5). It is worth mentioning that when the male patients aged 75 years or above were compared to the rest of the older adults, the true positive rate of MEWS's cut-off points can be as high as $100 \%$ for the male aged 75 years or above group, and $84.62 \%$ for the other group. This indicates that with our proposed cut-off scores, MEWS works very well in correctly identifying the true high-risk patients. We suggest that these cut-off points could potentially be used as a simple reference to rapidly identify the high-risk older adults in the near future.

\section{LIMITATION}

There are some limitations in this study. Firstly, it was a retrospective study conducted in a single center with relatively small sample size. Secondly, this study did not examine the younger population, but it may enable us to generalize the results to the other population. Thirdly, it would be interesting to explore how the heterogeneity in treatments over time would affects the effectiveness of MEWS and other scores. Statistically speaking, if such kind of data is available, more sophisticated models (e.g. logistic regression with mixed effects, or varying coefficient models) can be conducted to take into account possible heterogeneity in the data, and obtain better understandings for the utility of MEWS. Last but not least, although this study revealed 
282 that there is a significant association between MEWS and mortality in elderly patients with

283 COVID-19 upon admission, questions such as whether or not subsequent treatments are

284 associated with the reduction of MEWS, and whether or not the reduction of MEWS in

285 subsequent treatments is associated with lower mortality, are yet to be further investigated in

286 future studies.

287

288

\section{CONCLUSION}

Our Retrospective cohort study showed that, in terms of prognostic accuracy, MEWS performed

proven to be a promising tool for risk stratifications among the older adults COVID-19

population in this study. We have not only found MEWS to be an important variable for the risk,

but also identified the subpopulation consists of male COVID-19 patients aged 75 years or above

as the group with higher risk of mortality. Our analysis further suggested a cut-off point of

MEWS score 2.5 for male COVID-19 patients aged 75 years or above and a cut-off point of

MEWS score 3.5 for other older adults with COVID-19.

\section{ADDITIONAL INFORMATION AND DECLARATIONS}

298

299

300

301

302

\section{Funding}

This research did not receive any specific grant from funding agencies in the public, commercial, or not-for-profit sectors.

\section{Conflict of Interest Disclosures:}

All authors have completed the ICMJE Form for Disclosure of Potential Conflicts of Interest. 
303 Binyan Jiang was partially supported by an Alibaba (China) - Hong Kong Polytechnic University

304 Collaborative Research project.

305 Author Contributions

306 Lei Chen had full access to all of the data in the study and take responsibility for the integrity of

307 the data and the accuracy of the data analysis. Lichun Wang and Qingquan Lv contributed

308 equally and share first authorship. Concept and design: Lei Chen, Lichun Wang, Qingquan Lv,

309 Xiaofei Zhang. Acquisition, analysis, or interpretation of data: Lei Chen, Qingquan Lv, Binyan

310 Jiang, Xiaofei Zhang. Drafting of the manuscript: Lichun Wang, Binyan Jiang, Xiaofei Zhang,

311 En he Liu, Chaoxing Xiao. Critical revision of the manuscript for important intellectual content:

312 Lei Chen, Chunhua Yang. Statistical analysis: Binyan Jiang, Xinyang Yu, Lichun Wang.

313 Administrative, technical, or material support: En he Liu, Chaoxing Xiao. Supervision: Lei

314 Chen.

315 Data Availability

316 The following information was supplied regarding data availability:

317 The raw measurements are available in the Supplemental File. 
319

320

321

322

323

324

325

326

327

328

329

330

331

332

333

334

335

336

337

338

339

340

341

342

343

344

345

346

347

348

349

350

351

352

353

354

355

356

357

358

\section{REFERENCES}

Asai N, Watanabe H, Shiota A, Kato H, Sakanashi D, Hagihara M, Koizumi Y, Yamagishi Y, Suematsu H, and Mikamo H. 2019. Efficacy and accuracy of qSOFA and SOFA scores as prognostic tools for community-acquired and healthcare-associated pneumonia. Int $J$ Infect Dis 84:89-96. 10.1016/j.ijid.2019.04.020

Chalmers JD, Mandal P, Singanayagam A, Akram AR, Choudhury G, Short PM, and Hill AT. 2011. Severity assessment tools to guide ICU admission in community-acquired pneumonia: systematic review and meta-analysis. Intensive Care Med 37:1409-1420. 10.1007/s00134-011-2261-x

Churpek MM, Carey KA, Dela Merced N, Prister J, Brofman J, and Edelson DP. 2019. Validation of Early Warning Scores at Two Long-Term Acute Care Hospitals. Crit Care Med 47:e962-e965. 10.1097/ccm.0000000000004026

Dremsizov T, Clermont G, Kellum JA, Kalassian KG, Fine MJ, and Angus DC. 2006. Severe sepsis in community-acquired pneumonia: when does it happen, and do systemic inflammatory response syndrome criteria help predict course? Chest 129:968-978. 10.1378/chest.129.4.968

Fullerton JN, Price CL, Silvey NE, Brace SJ, and Perkins GD. 2012. Is the Modified Early Warning Score (MEWS) superior to clinician judgement in detecting critical illness in the pre-hospital environment? Resuscitation 83:557-562. 10.1016/j.resuscitation.2012.01.004

Goodacre S, Challen K, Wilson R, and Campbell M. 2010. Evaluation of triage methods used to select patients with suspected pandemic influenza for hospital admission: cohort study. Health Technol Assess 14:173-236. 10.3310/hta14460-03

Jo S, Jeong T, Lee JB, Jin Y, Yoon J, and Park B. 2016. Validation of modified early warning score using serum lactate level in community-acquired pneumonia patients. The National Early Warning Score-Lactate score. Am J Emerg Med 34:536-541. 10.1016/j.ajem.2015.12.067

Kao KC, Chang KW, Chan MC, Liang SJ, Chien YC, Hu HC, Chiu LC, Chen WC, Fang WF, Chen YM, Sheu CC, Tsai MJ, Perng WC, Peng CK, Wu CL, Wang HC, and Yang KY. 2018. Predictors of survival in patients with influenza pneumonia-related severe acute respiratory distress syndrome treated with prone positioning. Ann Intensive Care 8:94. 10.1186/s13613-018-0440-4

Kim HL, Kim MA, Park KT, Choi DJ, Han S, Jeon ES, Cho MC, Kim JJ, Yoo BS, Shin MS, Kang SM, Chae SC, Ryu KH, and Kor HFR. 2019. Gender difference in the impact of coexisting diabetes mellitus on long-term clinical outcome in people with heart failure: a report from the Korean Heart Failure Registry. Diabet Med. 10.1111/dme.14059

Kramer AA, Sebat F, and Lissauer M. 2019. A review of early warning systems for prompt detection of patients at risk for clinical decline. J Trauma Acute Care Surg 87:S67-s73. 10.1097/ta.0000000000002197

Liu K, Chen Y, Lin R, and Han K. 2020. Clinical features of COVID-19 in elderly patients: A comparison with young and middle-aged patients. $J$ Infect. 10.1016/j.jinf.2020.03.005 
Ning P, Zheng Y, Luo Q, Liu X, Kang Y, Zhang Y, Zhang R, Xu Y, Yang D, Xi W, Wang K, Chen Y, An S, and Gao Z. 2018. Metabolic profiles in community-acquired pneumonia: developing assessment tools for disease severity. Crit Care 22:130. 10.1186/s13054-0182049-2

Onder G, Rezza G, and Brusaferro S. 2020. Case-Fatality Rate and Characteristics of Patients Dying in Relation to COVID-19 in Italy. Jama. 10.1001/jama.2020.4683 \%J JAMA

Reini K, Fredrikson M, and Oscarsson A. 2012. The prognostic value of the Modified Early Warning Score in critically ill patients: a prospective, observational study. Eur J Anaesthesiol 29:152-157. 10.1097/EJA.0b013e32835032d8

Salottolo K, Carrick M, Johnson J, Gamber M, and Bar-Or D. 2017. A retrospective cohort study of the utility of the modified early warning score for interfacility transfer of patients with traumatic injury. BMJ Open 7:e016143. 10.1136/bmjopen-2017-016143

Sikka P, Jaafar WM, Bozkanat E, and El-Solh AA. 2000. A comparison of severity of illness scoring systems for elderly patients with severe pneumonia. Intensive Care Med 26:18031810. 10.1007/s001340000719

Verity R, Okell LC, Dorigatti I, Winskill P, Whittaker C, Imai N, Cuomo-Dannenburg G, Thompson H, Walker PGT, Fu H, Dighe A, Griffin JT, Baguelin M, Bhatia S, Boonyasiri A, Cori A, Cucunubá Z, FitzJohn R, Gaythorpe K, Green W, Hamlet A, Hinsley W, Laydon D, Nedjati-Gilani G, Riley S, van Elsland S, Volz E, Wang H, Wang Y, Xi X, Donnelly CA, Ghani AC, and Ferguson NM. 2020. Estimates of the severity of coronavirus disease 2019: a model-based analysis. The Lancet Infectious Diseases. https://doi.org/10.1016/S1473-3099(20)30243-7

Wang CC, Wu CK, Tsai ML, Lee CM, Huang WC, Chou HH, Huang JL, Chi NH, Yen HW, Tzeng BH, Chang WT, Chang HY, Wang CH, Lu YY, Tsai JP, Su CH, Cherng WJ, Yin WH, Tsai CT, Wu YW, Lin JL, and Hwang JJ. 2019. 2019 Focused Update of the Guidelines of the Taiwan Society of Cardiology for the Diagnosis and Treatment of Heart Failure. Acta Cardiol Sin 35:244-283. 10.6515/ACS.201905_35(3).20190422A

Wang D, Hu B, Hu C, Zhu F, Liu X, Zhang J, Wang B, Xiang H, Cheng Z, Xiong Y, Zhao Y, Li Y, Wang X, and Peng Z. 2020. Clinical Characteristics of 138 Hospitalized Patients With 2019 Novel Coronavirus-Infected Pneumonia in Wuhan, China. Jama. 10.1001/jama.2020.1585

Wu Z, and McGoogan JM. 2020. Characteristics of and Important Lessons From the Coronavirus Disease 2019 (COVID-19) Outbreak in China: Summary of a Report of 72314 Cases From the Chinese Center for Disease Control and Prevention. Jama. 10.1001/jama.2020.2648

Yang X, Yu Y, Xu J, Shu H, Xia J, Liu H, Wu Y, Zhang L, Yu Z, Fang M, Yu T, Wang Y, Pan S, Zou X, Yuan S, and Shang Y. 2020. Clinical course and outcomes of critically ill patients with SARS-CoV-2 pneumonia in Wuhan, China: a single-centered, retrospective, observational study. Lancet Respir Med. 10.1016/S2213-2600(20)30079-5

Yu M, Huang B, Liu P, Wang A, Ding W, Zhai Y, Huang Y, Zhong Y, Jian Z, Huang H, Hou B, and Xiong D. 2019. Detection of deteriorating patients after Whipple surgery by a 
400

401

402

403

404

405

modified early warning score (MEWS). Ann Transl Med 7:574. 10.21037/atm.2019.09.24

Zhou F, Yu T, Du R, Fan G, Liu Y, Liu Z, Xiang J, Wang Y, Song B, Gu X, Guan L, Wei Y, Li H, Wu X, Xu J, Tu S, Zhang Y, Chen H, and Cao B. 2020. Clinical course and risk factors for mortality of adult inpatients with COVID-19 in Wuhan, China: a retrospective cohort study. Lancet 395:1054-1062. 10.1016/S0140-6736(20)30566-3 
406

407

408

409

410

411

412

413

414

415

416

417

418

419

420

421

422

\section{LEGENDS}

\section{Figure legends}

Figure 1: The Proportion of Survivors and Non- Survivors in Older Adults with COVID-19 by

MEWS, APACHE II, SOFA, PSI, CURB-65, qSOFA, SIRS and age.

Figure 2: Area Under the Receiver Operating Characteristic Curve to Discriminate in-hospital

Mortality for MEWS, APACHE II, SOFA, PSI, CURB-65, SISR, qSOFA.

\section{Supplemental material legends}

Supplementary Text S1: Diagnosis and clinical classification of COVID-19-According to the Chinese Clinical Guidance for COVID-19 Pneumonia Diagnosis and Treatment 5th edition Supplementary Text S2: APACHE II score, CURB-65 score, PSI score, SOFA score, MEWS score, qSOFA score, and SIRS Criteria.

Supplementary Table S1: AUROC of MEWS in COVID-19 Patients between Male Patients Age $\geq 75$ Years and the Others.

Supplementary Figure S1: Distribution of patients with COVID-19 in Older Adults by MEWS, APACHE II, SOFA, PSI, CURB-65, qSOFA and SIRS.

Supplementary Figure S2: The Age-adjusted Receiver Operating Characteristic Curve by MEWS, APACHE II, SOFA, PSI, CURB-65, SISR, qSOFA. 


\section{Figure 1}

The Proportion of Survivors and Non- Survivors in Older Adults with COVID-19 by MEWS, APACHE II, SOFA, PSI, CURB-65, qSOFA, SIRS and age.

The proportion of survivors and Non- Survivors in older adults with COVID-19 by MEWS (Fig 1.A), APACHE II (Fig 1.B), SOFA ( Fig 1.C), PSI ( Fig 1.D), CURB-65 ( Fig 1.E), qSOFA ( Fig 1.F), SIRS ( Fig 1.G) and age ( Fig 1.H)
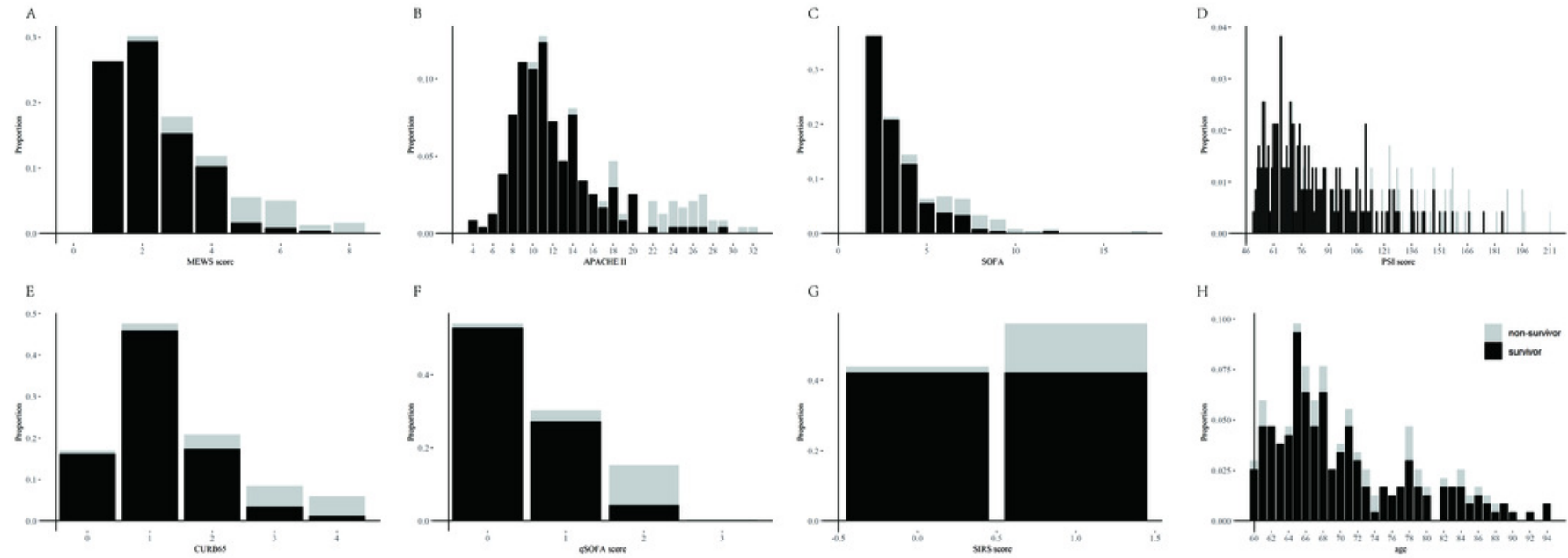
Figure 2

Area Under the Receiver Operating Characteristic Curve to Discriminate in-hospital Mortality for MEWS, APACHE II, SOFA, PSI, CURB-65, SISR, qSOFA.

ROC analysis suggested that the capacity of MEWS in predicting in-hospital mortality was as good as the APACHE II, SOFA, PSI and qSOFA, but was significantly higher than SIRS and CURB-65. 
ROC

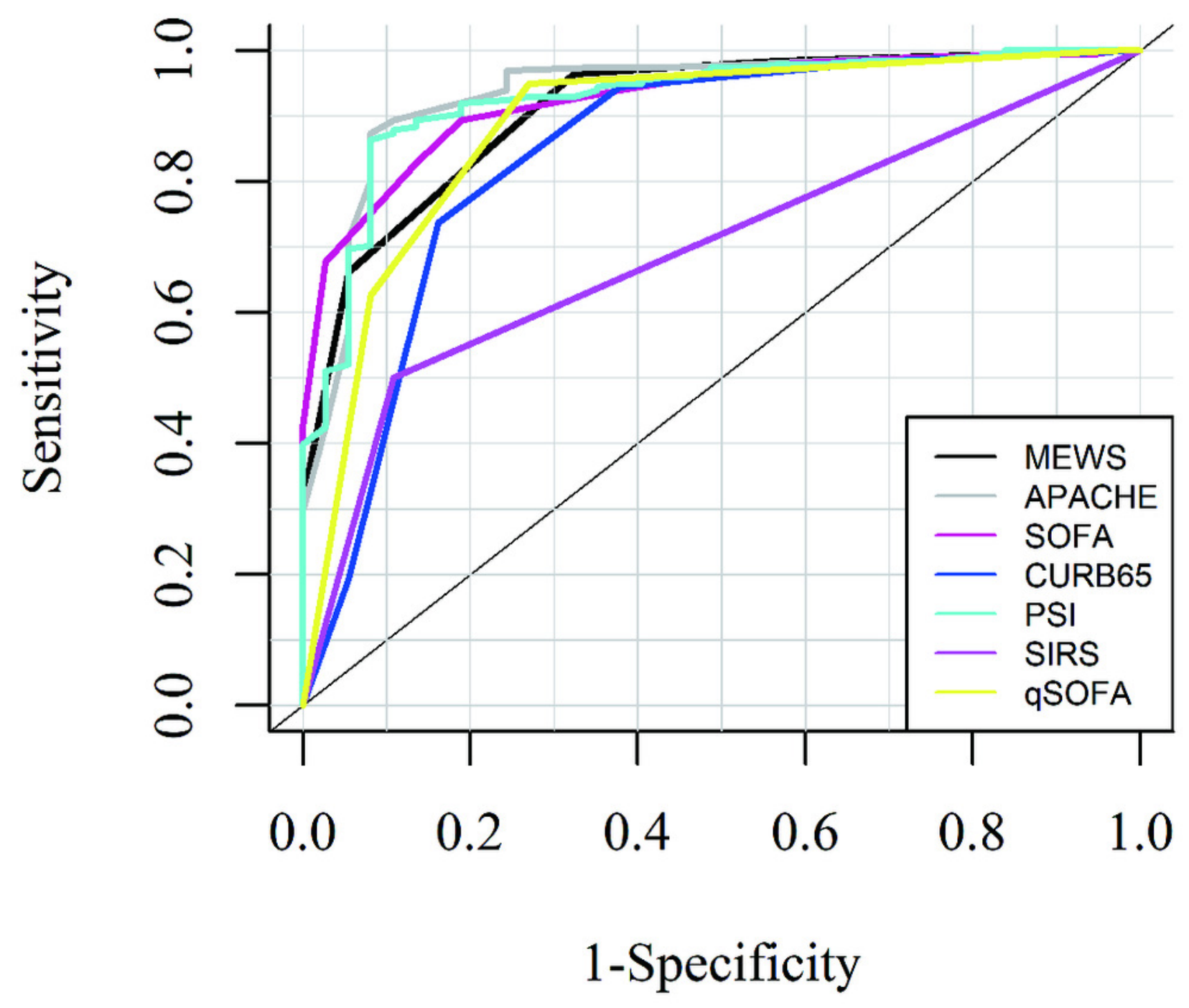




\section{Table $\mathbf{1}$ (on next page)}

Patient Characteristics and Outcome

APACHE II, Acute Physiology and Chronic Health Evaluation II; SOFA, Sequential Organ

Function Assessment; qSOFA: quick Sequential Organ Function Assessment; PSI, Pneumonia Severity Index; CURB-65, The Combination of Confusion, Urea, Respiratory Rate, Blood Pressure, and Age $\geq 65$; MEWS, Modified Early Warning Score; SIRS, Systemic Inflammatory Response Syndrome 
1 Table 1 Patient Characteristics and Outcome

\begin{tabular}{|c|c|c|c|c|}
\hline & All patients & Survivors & Non-survivors & $P$ \\
\hline Number of patients & 235 & 198 & 37 & \\
\hline Age, mean (SD), y & $70.6(8.0)$ & $70.17(9.9)$ & $72.95(8.1)$ & 0.530 \\
\hline Male, No. (\%) & $131(55.7)$ & $104(52.5)$ & $27(73.0)$ & 0.030 \\
\hline \multicolumn{5}{|l|}{ Co-morbidity No. (\%) } \\
\hline Hypertension & $103(43.8)$ & $89(45.0)$ & $14(37.8)$ & 0.474 \\
\hline Coronary heart disease & $49(20.9)$ & $41(20.7)$ & $8(21.6)$ & $>.999$ \\
\hline Diabetes mellitus & $62(26.4)$ & $52(26.3)$ & $10(27.0)$ & $>.999$ \\
\hline Chronic obstructive pulmonary & $31(13.2)$ & $29(14.7)$ & $3(8.1)$ & 0.433 \\
\hline \multicolumn{5}{|l|}{ disease } \\
\hline Cerebrovascular disease & $19(8.1)$ & $16(8.0)$ & $3(8.1)$ & $>.999$ \\
\hline Cancer & $8(3.4)$ & $7(3.5)$ & $1(2.7)$ & $>.999$ \\
\hline Others & $22(9.4)$ & $21(10.6)$ & $1(2.7)$ & 0.215 \\
\hline None & $67(28.5)$ & $57(28.8)$ & $12(32.4)$ & $>.999$ \\
\hline \multicolumn{5}{|l|}{ Severity of Illness No. (\%) } \\
\hline mild & $98(41.7)$ & $97(49.0)$ & $1(2.7)$ & $<0.001$ \\
\hline moderate & $48(20.4)$ & $47(23.7)$ & $1(2.7)$ & 0.002 \\
\hline severe & $89(37.9)$ & $54(27.3)$ & $35(94.6)$ & $<0.001$ \\
\hline \multicolumn{5}{|l|}{ Scores on Admission, median (IQR), } \\
\hline APACHE II & $12(9,17)$ & $11(9,14)$ & $24(20,27)$ & $<0.001$ \\
\hline SOFA & $3(2,5)$ & $3(2,4)$ & $7(6,9)$ & $<0.001$ \\
\hline PSI & $82(65,114)$ & $75(63,100)$ & $152(128,166)$ & $<0.001$ \\
\hline CURB65 & $1(1,2)$ & $1(1,2)$ & $3(2,4)$ & $<0.001$ \\
\hline MEWS & $2(1,4)$ & $2(1,3)$ & $5(4,6)$ & $<0.001$ \\
\hline qSOFA & $0(0,1)$ & $0(0,1)$ & $2(1,2)$ & $<0.001$ \\
\hline SIRS criteria $\geq 2$ No. $(\%)$ & $132(56.2)$ & $99(50)$ & $33(89.19)$ & $<.0001$ \\
\hline
\end{tabular}


Outcome

Hospital mortality (primary

outcome), No. (\%)

Hospital length of stay, median

(IQR), d
$37(15.8)$

$13(6,23)$
0

37 (100)

$<0.001$

$15(8,24)$

$5.5(2.0,9.3)$

$<0.001$

APACHE II, Acute Physiology and Chronic Health Evaluation II; SOFA, Sequential Organ Function Assessment;

3 qSOFA: quick Sequential Organ Function Assessment; PSI, Pneumonia Severity Index; CURB-65, The

4 Combination of Confusion, Urea, Respiratory Rate, Blood Pressure, and Age $\geq 65$; MEWS, Modified Early Warning

5 Score; SIRS, Systemic Inflammatory Response Syndrome 


\section{Table 2 (on next page)}

Prognostic Accuracy of Difference Score in Predicting the In-Hospital Mortality and the Difference with AUROC of MEWS

APACHE II, Acute Physiology and Chronic Health Evaluation II; SOFA, Sequential Organ Function Assessment ; qSOFA: quick Sequential Organ Function Assessment; PSI, Pneumonia Severity Index; CURB-65, The Combination of Confusion, Urea, Respiratory Rate, Blood Pressure, and Age $\geq 65$; MEWS, Modified Early Warning Score; SIRS, Systemic Inflammatory Response Syndrome; AUROC, area under the receiver operating characteristic curve; SEN, sensitivity; SPE, specificity; NPV, negative predictive value; PPV, positive predictive value. 
1 Table 2 Prognostic Accuracy of Difference Score in Predicting the In-Hospital Mortality and the Difference with 2 AUROC of MEWS

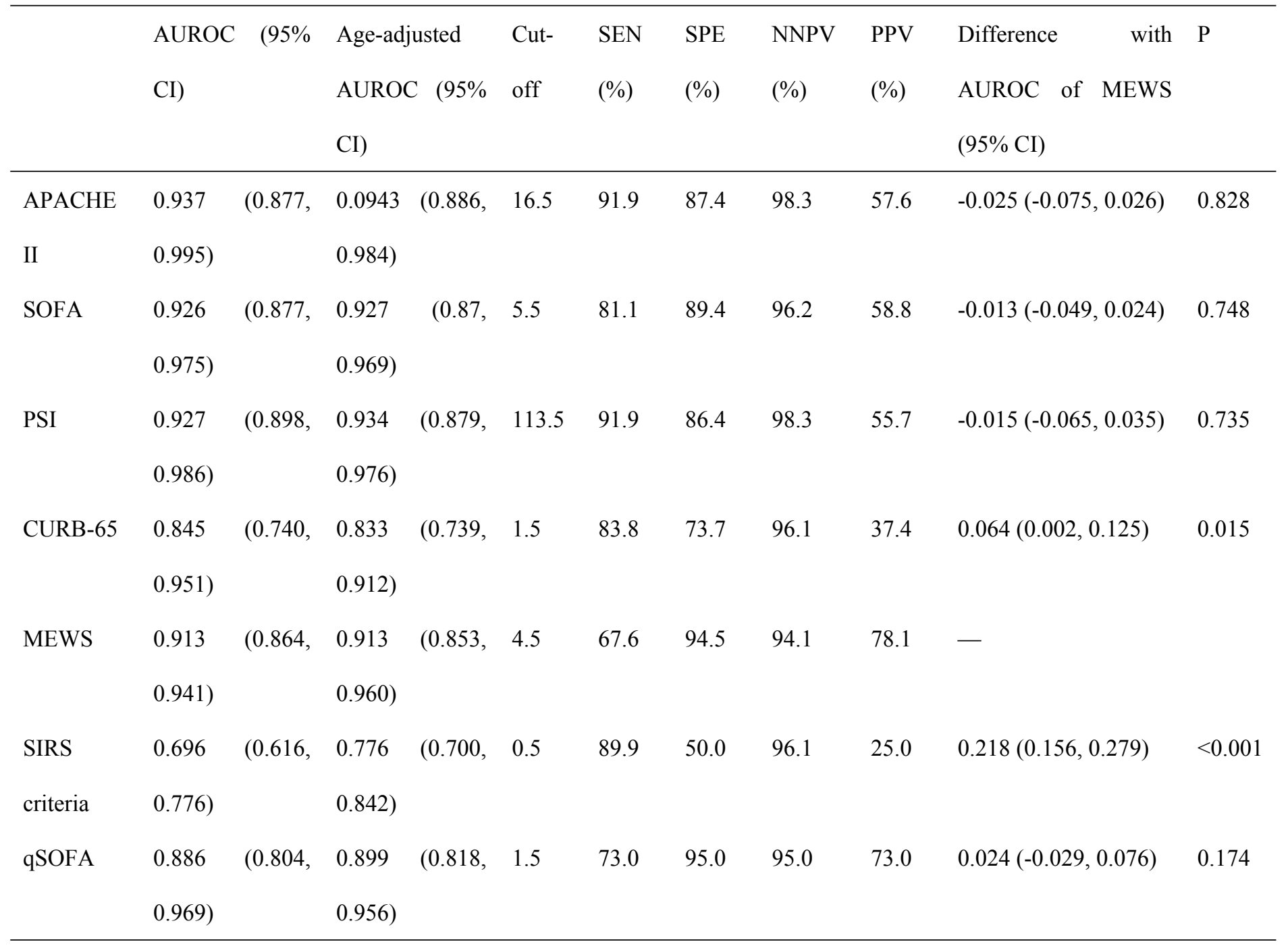

3 APACHE II, Acute Physiology and Chronic Health Evaluation II; SOFA, Sequential Organ Function Assessment;

4 qSOFA: quick Sequential Organ Function Assessment; PSI, Pneumonia Severity Index; CURB-65, The Combination

5 of Confusion, Urea, Respiratory Rate, Blood Pressure, and Age $\geq 65$; MEWS, Modified Early Warning Score; SIRS,

6 Systemic Inflammatory Response Syndrome; AUROC, area under the receiver operating characteristic curve; SEN,

7 sensitivity; SPE, specificity; NPV, negative predictive value; PPV, positive predictive value.

8 


\section{Table 3 (on next page)}

Logistic Regression Models of the Age Group, Gender and MEWS

MEWS, Modified Early Warning Score. Model 1: MEWS; Model 2: Gender*Age +MEWS; Model

3: Age +MEWS; Model 4: Gender +MEWS; Model 5: Age + Gender*Age + MEWS; Model

6:Age + Gender*Age + MEWS + Gender; Model 7๑Age + Gender + MEWS; Model 8:

Gender*Age + Gender + MEWS. Logistic regression models were developed against MEWS, age groups ("1" for 75 years old or above, and "0" for 60-74 years old), gender (" 1 " for male and " 0 " for female), and the interaction between age group and gender (age*gender). 
1 Table 3 Logistic Regression Models of the Age Group, Gender and MEWS

\begin{tabular}{|c|c|c|c|c|c|c|c|c|c|c|c|c|c|}
\hline \multirow[t]{3}{*}{ Model } & \multirow[t]{3}{*}{ AIC } & \multicolumn{4}{|c|}{ Estimated Coefficients } & \multicolumn{5}{|c|}{ Standard Error } & \multicolumn{3}{|c|}{$\mathrm{P}$} \\
\hline & & Age & Gen & Gender & MEW & Age & Gen & Gender* & ME & Age & Gende & Gender* & MEWS \\
\hline & & & der & $*$ Age & $\mathrm{S}$ & & der & Age & WS & & $\mathrm{r}$ & Age & \\
\hline 1 & 118.01 & - & - & - & 1.24 & - & - & - & 0.18 & - & - & - & $<0.001$ \\
\hline 2 & 116.03 & - & - & 1.16 & 1.24 & - & - & 0.58 & 0.18 & - & - & 0.044 & $<0.001$ \\
\hline 3 & 117.68 & 0.79 & - & - & 1.23 & 0.51 & - & - & 0.18 & 0.125 & - & - & $<0.001$ \\
\hline 4 & 116.54 & - & 0.96 & - & 1.24 & - & 0.53 & - & 0.19 & - & 0.071 & - & $<0.001$ \\
\hline 5 & 118.03 & 0.04 & - & 1.13 & 1.24 & 0.82 & - & 0.91 & 0.18 & 0.964 & - & 0.214 & $<0.001$ \\
\hline 6 & 118.52 & 0.50 & 0.80 & 0.34 & 1.24 & 0.92 & 0.66 & 1.12 & 0.19 & 0.584 & 0.228 & 0.764 & $<0.001$ \\
\hline 7 & 116.61 & 0.73 & 0.91 & - & 1.24 & 0.52 & 0.54 & - & 0.19 & 0.163 & 0.089 & - & $<0.001$ \\
\hline 8 & 116.81 & - & 0.65 & 0.84 & 1.24 & - & 0.59 & 0.64 & 0.19 & - & 0.272 & 0.189 & $<0.001$ \\
\hline
\end{tabular}

2 MEWS, Modified Early Warning Score. Model 1: MEWS; Model 2: Gender*Age +MEWS; Model 3: Age +MEWS;

3 Model 4: Gender +MEWS; Model 5: Age + Gender *Age + MEWS; Model 6:Age + Gender*Age + MEWS + Gender;

4 Model 7:Age + Gender + MEWS; Model 8: Gender*Age + Gender + MEWS. Logistic regression models were

5 developed against MEWS, age groups ("1" for 75 years old or above, and " 0 ” for 60-74 years old), gender ("1" for

6 male and "0" for female), and the interaction between age group and gender (age*gender).

7

8

9 